\title{
Erratum to: Representation Formulas of Curves in a Two- and Three-Dimensional Lightlike Cone
}

Huili Liu and Qingxian Meng

\section{Erratum to: Results. Math. (2011) 59:437-451 DOI 10.1007/s00025-011-0108-y}

In the original publication of the article, Case 3 in Theorem 2.4 has been inadvertently missed out. The complete Theorem 2.4 is given below.

Theorem 2.4. Let $x: \mathbf{I} \rightarrow \mathbb{Q}^{2} \subset \mathbb{E}_{1}^{3}$ be a spacelike curve in $\mathbb{Q}^{2}$ with arc length parameter $s$ and structure function $f(s)$. If the curve $x$ is a non planar helix, the structure function $f(s)$ satisfies

$$
\left[\left(\log f_{s}\right)_{s}\right]^{2}-2\left(\log f_{s}\right)_{s s}=2 \kappa(s)=a(s+b)^{-2}
$$

and can be written as (by an appropriate parameter transformation)

Case 1. $f(s)=s^{c}$ or $f(s)=s^{-c}$, for $c \neq 0, \pm 1$ and $a=c^{2}-1$;

Case 2. $f(s)=\frac{c}{\log s}$ or $f(s)=\frac{\log s}{c}$, for $c \neq 0$ and $a=-1$;

Case 3. $f(s)=\frac{2}{c} \tan \left(\frac{c}{2} \log s\right)$ or $f(s)=-\frac{2}{c} \tan ^{-1}\left(\frac{c}{2} \log s\right)$, for $c \neq 0$ and $a+1=-c^{2}$. 
Huili Liu and Qingxian Meng

Department of Mathematics

Northeastern University

Shenyang 110004

People's Republic of China

e-mail: liuhl@mail.neu.edu.cn;

mengqingxian8@126. com

URL: http://faculty.neu.edu.cn/liuhl/ 